\title{
EINE TRENDWENDE IST NICHT ZU ERWARTEN
}

Konstantin Beck

Prof. Dr. oec., Gesundheitsökonom, Universität Luzern

\begin{abstract}
Während der letzten 100 Jahre stiegen die Gesundheitskosten kontinuierlich und exponentiell. Als wichtigste Treiber lassen sich die rasante technologische Entwicklung zusammen mit einer dank den Sozialversicherungen gestärkten Kaufkraft der Nachfrage identifizieren. Solange Innovationskraft und Finanzierungsfähigkeit aufrechterhalten bleiben, spricht viel dafür, dass die Kostenentwicklung auch in Zukunft ähnlich verlaufen wird.
\end{abstract}

Das Wort "Gesundheitskosten» ist in der Öffentlichkeit erstaunlich negativ behaftet. Wieso ist das so? Erwarten die Menschen, dass die medizinische Versorgung gratis sei? Oder glauben sie, der medizinisch-technische Fortschritt falle ganz einfach vom Himmel? Kosten entstehen dort, wo neve medizinisch-technische Möglichkeiten ausgeschöpft werden, dies wiederum führt zu einem besseren Gesundheitszustand der Bevölkerung. Wenn Ökonomen von Kosten sprechen, dann geht es in der Regel um Wirtschaftlichkeit und nicht um simple Kostenvermeidung. Sie fragen sich, ob mit den verfügbaren Mitteln noch mehr Menschen noch besser geholfen werden könnte, oder ob mit weniger Mitteln denselben Menschen genau gleich gut geholfen werden kann.

\section{Der Blick zurück}

Die Gesundheitskosten der Vergangenheit sind bekannt. So können wir beispielsweise die Daten für die staatlich anerkannte soziale Krankenpflege-Grundversicherung bis 1920 zurückverfolgen.

1920 versursachte die Grundversicherung Kosten von Fr. 15.24 pro Kopf und Jahr. Demgegenüber liegen die Kosten im Jahr 2015 bei Fr. 4015.95. Das entspricht zwar rein buchhalterisch einem Anstieg von $26^{\prime} 000 \%$. Diese Prozentgrösse ist für sich allein genommen jedoch nichtssagend. So war die Kaufkraft des Franken im Jahr 1915 eine ganz andere, die Löhne unterscheiden sich eklatant, und vor allem die Versicherungsdeckungen von damals und heute weisen kaum noch Schnittmengen auf. Die Kosten von 2015 decken zum grössten Teil Behandlungen ab, die vor hundert Jahren gar nicht vorstellbar waren.
Interessanter sind daher zwei Fragen: Wie entwickelten sich die Kosten während der letzten 100 Jahre? Und was waren die treibenden Kräfte? Betrachtet man die Fünf-Jahres-Daten von 1920 bis 2015 in der Tabelle, so fällt zunächst auf, dass sie kontinuierlich ansteigen. Einzig 1940 nehmen die Kosten (geringfügig) ab. Dies dürfte wahrscheinlich ein Nebeneffekt der damaligen Generalmobilmachung gewesen sein. Auch die grosse Wirtschaftskrise von 1929 schlägt mit dem zweittiefsten

Kostenanstieg in 100 Jahren zu Buche. Aber noch vor Ende des Krieges findet die Zeitreihe auf ihren Wachstumspfad zurück.

Zwei Ereignisse sind erwähnenswert: So wurde das Krankenversicherungsgesetz 1964 teil- und 1996 totalrevidiert. Beide Ereignisse sind in der Zeitreihe nicht erkennbar, auf alle Fälle nicht als kostendämpfende Effekte. Ganz im Gegenteil, setz† sich doch ab 1950 ein exponentielles Wachstum durch, das ungebremst bis zum Jahr 2015 anhält.

Basierend auf dieser Zeitreihe publizierte ich 2004 eine recht simple exponentielle Regression, die den Verlauf bis ins Jahr 2000 erstaunlich gut abbilden konnte $\left(R^{2}=99,6 \%\right)$ [1]. Noch erstaunlicher bei dieser Berechnung war die Treffgenauigkeit ihrer Prognosen. Eine Fortschreibung dieses exponentiellen Wachstums liefert eine auf 5\% genaue Prognose der Kosten von 2015. Es überrascht, dass sich die Entwicklung der Gesundheitskosten mit einer so einfachen Extrapolation, die ausser der Trendvariablen keinerlei inhaltlichen Input verwendet, so genau vorhersagen lässt. Weder die Umstellung der Spitalfinanzierung noch das markante Wachstum von Managed Care hatte einen erkennbaren Einfluss auf den Kostenverlauf. Die Kosten erweisen sich als erstaunlich «Politik-resistent». 
Tabelle 1: Bruttoleistungen pro Kopf der Krankenpflege-Grundversicherung von CSS-Versicherten 1920 bis 2015 in Franken.

\begin{tabular}{llllll} 
Jahr & Kosten/Kopf & Anstieg & Jahr & Kosten/Kopf & Anstieg \\
\hline 1920 & 15.24 & $*$ & 1975 & 426.69 & $94 \%$ \\
\hline 1925 & 22.80 & $50 \%$ & 1980 & 552.89 & $30 \%$ \\
\hline 1930 & 26.19 & $15 \%$ & 1985 & 842.15 & $52 \%$ \\
\hline 1935 & 33.13 & $26 \%$ & 1990 & $1^{\prime} 137.56$ & $35 \%$ \\
\hline 1940 & 31.32 & $-5 \%$ & 1995 & $1^{\prime} 433.87$ & $26 \%$ \\
\hline 1945 & 41.71 & $33 \%$ & 2000 & $2^{\prime} 015.64$ & $41 \%$ \\
\hline 1950 & 55.40 & $33 \%$ & 2005 & $2^{\prime} 714.16$ & $35 \%$ \\
\hline 1955 & 70.73 & $28 \%$ & 2010 & $3^{\prime} 320.59$ & $22 \%$ \\
\hline 1960 & 85.29 & $21 \%$ & 2015 & $4^{\prime} 015.95$ & $21 \%$ \\
\hline 1965 & 121.69 & $43 \%$ & & & \\
\hline 1970 & 220.15 & $81 \%$ & 2120 & $60^{\prime} 000.00$ & $*$ \\
\hline
\end{tabular}

*missing value

Quelle: Jahresberichte der Christlich Sozialen Kranken- und Unfallversicherung respektive der CSS. Ab 2000: Angaben der CSS für Statistiken des BSV und BAG.

\section{Die treibenden Faktoren}

Welches sind nun aber die kostentreibenden Faktoren? Regelmässig wird an erster Stelle die demographische Alterung als Grund angeführt. Diese These ist nicht nur in der Literatur heftig umstritten, auch die Daten sprechen dagegen. So setzt die starke demographische Verschiebung bei den Erwachsenen erst in den 1980er Jahren ein. Somit kann der exponentielle Kostenanstieg zwischen 1920 und 1980 nicht mit der Alterung der Bevölkerung erklärt werden. Zudem schwächt sich der «rein demographische Effekt» seit 2010 bereits wieder ab [2].

Auch das Bevölkerungswachstum der Schweiz erklärt nur einen knappen Viertel des Kostenanstiegs. Als Kostentreiber verbleiben somit die steigende Nachfrage nach medizinischen Leistungen und der technische Fortschritt (zusammen knapp drei Viertel des Kostenanstiegs) [3]. Technischer Fortschritt in der Medizin umfasst die einleitend erwähnten immensen qualitativen und quantitativen medizinischen Verbesserungen von 1920 bis heute. Er muss sich üblicherweise auf Märkten mit Nachfrage und Angebot bewähren. Dabei kann es vorkommen, dass sich eine Innovation nicht durchsetzt. Im Gesundheitswesen, wo die Patienten praktisch nie die vollen Kosten des Fortschritts bezahlen müssen, setzen sich aber auch Innovationen durch, die in anderen Märkten durchfallen würden. Und noch etwas anderes fällt auf: Rasanter Fortschritt bedeutet nicht automatisch steigende Preise. Eine entsprechende Entwicklung beobachten wir in der Telekommunikation und in der Informatik. Noch nie war Telefonieren so billig wie heute und noch nie musste für einen Taschenrechner so wenig bezahlt werden. Für den medizinischen Bereich ist jedoch kein vergleichbares Beispiel bekannt, bei dem technischer Fortschritt zu nachhaltig und substantiell tieferen Kosten geführt hätte. Das ist erstaunlich.

\section{Der Blick in die Zukunft}

Extrapoliert man die erwähnte Regressionsgleichung in die Zukunft, dann verdoppeln sich die Kosten bis ins Jahr 2035 und betragen in hundert Jahren rund Fr. 60'000 pro Kopf. Darin wären dann alle Innovationen der Zukunft enthalten. Und solange das BIP ebenfalls exponentiell steigt (und das tut es - mit Unterbrüchen - seit 1750), ist nicht damit zu rechnen, dass diese hohen Gesundheitskosten nicht auch finanziert werden könnten. Aber ist dieser kontinuierliche Anstieg wirklich plausibel? Dafür sprechen langfristige kulturelle Gründe.

Der mittelalterliche Mensch verfügte über vergleichsweise rudimentärste medizinische Kenntnisse, aber er war mental besser gewappnet, Krankheit und Schmerz zu ertragen. Die damalige Heilkunde war in erster Linie Anleitung zur gesunden Lebensführung, was ins Heute übertragen am ehesten dem Begriff «Prävention» entspricht [4]. Diese Rahmenbedingungen führten dazu, dass dem (grossen) Bedarf an medizinischer Pflege schlicht und einfach nicht entsprochen werden konnte. Mit dem Einsetzen der naturwissenschaftlichen Revolution verschoben sich die Gewichte. Je besser das Angebot der Reparaturmedizin, desto geringer die Bereitschaft und auch die Notwendigkeit, eine Krankheit auszuhalten [5]. Dazu kommt eine systematische finanzielle Stärkung der Nachfrage über Sozialversicherungen. Das hat wie immer zwei Seiten: Der Mensch wird immer ängstlicher, und vieles, was früher als erträglich galt, wird heute pathologisiert. Ob das einer Gesellschaft nur guttut, sei dahingestellt - im Zusammenhang mit Corona ist dazu eine intensive Debatte im Gang. Tatsache bleibt jedoch, dass diese kulturelle Verschiebung, so sie anhält, auch in Zukunft für eine wachsende Nachfrage nach Gesundheits-Dienstleistungen sorgen dürfte.

\section{Literatur}

1 Beck K. Risiko Krankenversicherung. Bern: Haupt Verlag; 2004. 1. Auflage, S. 107.

2 Steinmann L, Telser H. Gesundheitskosten in der alternden Gesellschaft. Zürich: NZZ Verlag; 2005.

3 Beck K. Dasselbe, dritte Auflage 2013.

4 Vgl. Schockenhoeff mit Verweisen auf Paracelsus, Hildegard von Bingen, Plato etc. (Schockenhoff E. Die Verantwortung für das eigene Leben: Gesundheit und Krankheit, in: Derselbe, Ethik des Lebens. Grundlagen und neue Herausforderungen. Freiburg im Breisgau: Herder; 2009. S. 296-342.

5 Ebenda. 